\title{
Boundary Exact Controllability of the Heat Equation in 1D by Strategic Actuators and a Linear Surjective Compact Operator
}

\author{
Cheikh Seck $^{1,2}$, Libasse Ane ${ }^{3}$, Abdoulaye Sène ${ }^{3}$ \\ ${ }^{1}$ Département de Mathématiques de la FASTEF ex ENS de l’Université Cheikh Anta Diop de Dakar, Sénégal \\ ${ }^{2}$ Laboratoire d'Analyse Numérique et d'Informatique (LANI) de l'UFR SAT de l'Université Gaston Berger de Saint-Louis, Sénégal \\ ${ }^{3}$ Département de mathématiques et d’Informatique de la Faculté des Sciences et Techniques de l'Université Cheikh Anta Diop de \\ Dakar, Sénégal \\ Email: cheikh5.seck@ucad.edu.sn,malibsstar@gmail.com, abdoulaye.sene@ucad.edu.sn
}

How to cite this paper: Seck, C., Ane, L. and Sène, A. (2020) Boundary Exact Controllability of the Heat Equation in $1 \mathrm{D}$ by Strategic Actuators and a Linear Surjective Compact Operator. Applied Mathematics, 11, 991-999.

https://doi.org/10.4236/am.2020.1110065

Received: September 1, 2020

Accepted: October 16, 2020

Published: October 19, 2020

Copyright $\odot 2020$ by author(s) and Scientific Research Publishing Inc. This work is licensed under the Creative Commons Attribution International License (CC BY 4.0).

http://creativecommons.org/licenses/by/4.0/

\begin{abstract}
In this paper we show a boundary result of controllability by a new approach using a linear, continuous and surjective operator built from the solution of the heat system. And, subsequently, the border exact controllability of the 1D heat equation through a compactness criterion and the use of strategic zone actuators were established.
\end{abstract}

\section{Keywords}

Control, Controllability, Estimations, Strategic Actuator

\section{Introduction}

These last years, the exact controllability of distributed systems has been significantly enhanced by J. L. Lions [1] [2] with the development of the Hilbert Uniqueness Methods (HUM). It is based essentially on the uniqueness properties of the homogeneous equation by a particular choice of controls, the construction of a Hilbert space and a continuous linear application of this Hilbert space in its dual which is, in fact, an isomorphism that establishes exact controllability.

For hyperbolic problems, this method yielded important results (Lions [3]); although when the controls are small support (Niane [4], Seck [5] [6]), it seems not very effective, likewise when for technical reasons the multiplier method does not give satisfactory results.

As for the parabolic equations, there are the results of Imanuvilov-Fursikov [7] and G. Lebeau-L. Robbiano [8] who proved with different methods but very 
technical and long, the exact control of the Heat equation.

Also, the harmonic method is inoperative also for this kind of equations.

In this work, to circumvent certain constraints related to estimates in G. Lebeau's work, we show that a new method which solves some of these difficulties. It is based Seck's work; on criteria of surjectivity of a continuous linear operator of a Hilbert space in another construct directly from the problem of exact border controllability.

The criteria are of two types:

1) A surjectivity criterion that is a consequence of the properties of uniqueness (J. L. Lions);

2) A criterion of compactness that derives from the parabolic nature of the operator or the regularity of the control;

In both cases, these criteria are easier to verify than those of the Lions HUM method.

This method which we call Boundary Exact Controllability by Surjectivity and Compactness opens wide perspectives to the theory of the exact controllability in general, as well as to the theory of the exact controllability by actuators strategic zones and allows for the parabolic equations, from Schrödinger, plates, linearized Navier-Stockes to solve many questions thus opening up many perspectives.

\section{Characterization of Exact Controllability}

Indeed, we have the following result of functional analysis (see J. L. Lions and Ramdani [9]) which will allow us to characterize the exact controllability of the heat equation.

For proof see also jeups 2012 Karim-Ramdani or J. L. Lions.

\subsection{Exact Controllability Reminders}

Let $I=] 0,1[$ an open interval of $\mathbb{R}$. We put A to the operator defined by:

$$
D(A)=\left\{u \in H_{0}^{1}(I) /-\frac{\mathrm{d}^{2} u}{\mathrm{~d} x^{2}} \in L^{2}(I)\right\} ; \forall u \in D(A), A u=-\frac{\mathrm{d}^{2} u}{\mathrm{~d} x^{2}}
$$

Lemma 1. Let $\mathrm{E}$ and $\mathrm{H}$ two Hilbert spaces and $\mathcal{L} \in \mathcal{L}(E, H)$. Then, $\mathcal{L}$ is surjective if and only if its adjoint $\mathcal{L}^{*}$ is bounded below, i.e. there exists a constant $C>0$ such that

$$
\left\|\mathcal{L}^{*}(\beta)\right\|_{H} \geq C\|\beta\|_{E}, \quad \forall \beta \in H .
$$

Lemma 2. The following assertions are equivalent:

1) The system (12) below is exactly controllable for $T>0$.

2) The operator $\mathcal{L}_{T}^{*}$ is inferiorly bounded, i.e. there exists $C_{T}>0$ such that

$$
\left\|\mathcal{L}_{T}^{*}\right\|_{L^{2}(] 0, T[, I)}=\left(\int_{0}^{T}\left\|\mathcal{L}_{T}^{*} \mathrm{e}^{s A} z_{0}\right\|^{2} \mathrm{~d} s\right)^{\frac{1}{2}} \geq C_{T}\left\|z_{0}\right\|, \quad \forall z_{0} \in I
$$

See Lions, El. Jai [10] [11] or also Ramdani-Karim jeups 2012. 
Definition 1. Let $\mathcal{L}$ the operator defined below. We will say that the system defined by (12) that it is exactly controllable in time $\mathrm{T}$ if and only if $\mathcal{L}$ is surjective.

\subsection{Preliminary Results of Controllability}

Definition 2. An integrable square function $\mu: I \subset \Omega \rightarrow \overline{\mathbb{R}}$ is called strategic if it satisfies, for all $\psi_{0} \in L^{2}(I)$, the solution $\psi^{+}$of heat equation

$$
\left\{\begin{array}{l}
\left.\psi^{+^{\prime}}(t, x)-\Delta \psi^{+}(t, x)=0 \text { in } Q_{T}=\right] 0,+\infty[\times I \\
\left.\gamma \psi^{+}(t, x)=0 \text { in } \Sigma_{T}=\right] 0,+\infty[\times \partial I \\
\psi^{+}(0)=\psi_{0} \text { in } I
\end{array}\right.
$$

satisfies:

$$
\forall t>0, \int_{I} \mu(x) \psi^{+}(t, x) \mathrm{d} x=0 \text { then } \psi_{0}=0
$$

\section{Remark}

1) It suffices that the relation (5) be checked over an interval $] 0, T$ [ so that it is true over ] $0,+\infty\left[\right.$, because of the analycity of $t \mapsto \int_{I} \mu(x) \psi^{+}(t, x) \mathrm{d} x$ on $\mathbb{R}_{+}^{*}$, Brezis [12].

2) Here $\Omega$ is an open bounded of $\mathbb{R}^{2}$, of regular border; $L^{2}(I)$ is, a priori, the state space and $T$ defines the time horizon considered for the exact controllability of the system (4).

Proposition 3. There are strategic actuators with support contained in any interval $] a, b[$ such that:

$$
0<a<b<1
$$

Proof. We can first notice that $\mu$ is strategic if and only if: $\forall k \in \mathbb{N}^{*}, \mu_{k} \neq 0$.

Let $a, b \in] 0,1\left[\right.$ such that $a<b$ and assume that: $\mu=\chi_{a, b[}$.

So, we have

$$
\begin{aligned}
\mu_{k} & =\int_{0}^{1} \chi_{] a, b[}(x) \sqrt{2} \sin (k \pi x) \mathrm{d} x \\
& =-\frac{\sqrt{2}}{k \pi}[\cos (k \pi b)-\cos (k \pi a)] \\
& =-\frac{\sqrt{2}}{k \pi} 2 \sin \left(\frac{k \pi(b-a)}{2}\right) \sin \left(\frac{k \pi(b+a)}{2}\right)
\end{aligned}
$$

We have $\mu_{k}=0$ if and only if

$$
\left\{\begin{array}{l}
\frac{k \pi(b-a)}{2}=l \pi, \quad l \in \mathbb{Z} \\
\text { or } \\
\frac{k \pi(b+a)}{2}=r \pi, \quad r \in \mathbb{Z}
\end{array}\right.
$$

Therefore, for $\mu_{k} \neq 0$ it is enough that:

$$
b-a \notin \mathbb{Q} \text { and } b+a \notin \mathbb{Q} .
$$

So, if we take $a \in \mathbb{Q}$ and $b=a+r$ where $r \notin \mathbb{Q}$ then $\mu=\chi_{] a, b[}$ is strategic. 
Remark. Of course, other strategic actuators can be built without difficulty, see also El. Jai.

We define the Hilbert spaces that follow:

$$
\forall T>0, G_{T}=\left\{\mu=\sum_{k=1}^{+\infty} \mu_{k} w_{k} / \sum_{k=1}^{+\infty} \mu_{k}^{2} \mathrm{e}^{2 \lambda_{k} T}<+\infty\right\}
$$

We equip $G_{T}$ with the following scalar product $(,)_{G_{T}}$ :

$$
(x, y)_{G_{T}}=\sum_{k=1}^{\infty} x_{k} y_{k} \mathrm{e}^{2 \lambda_{k} T}
$$

and, of the norm $\|\cdot\|_{G_{T}}$.

We know that $G_{T}$ is a Hilbert space; its dual is defined by:

$$
\forall T>0, G_{T}^{*}=\left\{\mu=\sum_{k=1}^{+\infty} \mu_{k} w_{k} / \sum_{k=1}^{+\infty} \mu_{k}^{2} \mathrm{e}^{-2 \lambda_{k} T}<+\infty\right\}
$$

We equip $G_{T}^{*}$ with the scalar product

$$
(x, y)_{G_{T}^{*}}=\sum_{k=1}^{\infty} x_{k} y_{k} \mathrm{e}^{-2 \lambda_{k} T}
$$

and, of the norm $\|\cdot\|_{G_{T}^{*}}$.

We define the duality hook for $x \in G_{T}^{*}, y \in G_{T}$ by

$$
\langle x, y\rangle_{G_{T}^{*} G_{T}}=\sum_{k=1}^{\infty} x_{k} y_{k}
$$

Let $\psi_{0} \in G_{T}^{*}$, we notice $\psi^{+}$the solution of the following heat equation

$$
\left\{\begin{array}{l}
\left.\psi^{+^{\prime}}(t, x)-\Delta \psi^{+}(t, x)=0 \text { in }\right] 0, \infty[\times I \\
\left.\psi^{+}(t, 0)=\psi^{+}(t, 1)=0 \text { in }\right] 0, \infty[ \\
\psi^{+}(0, x)=\psi_{0}(x) \text { in } I
\end{array}\right.
$$

Let $\psi_{0} \in G_{T}$, we notice $\psi^{-}$the solution of

$$
\left\{\begin{array}{l}
\left.\psi^{-{ }^{-}}(t, x)-\Delta \psi^{-}(t, x)=0 \text { in }\right] 0, \infty[\times I \\
\left.\psi^{-}(t, 0)=\psi^{-}(t, 1)=0 \text { in }\right] 0, \infty[ \\
\psi^{-}(0, x)=\psi_{0}(x) \text { in } I
\end{array}\right.
$$

\section{Main Result of Boundary Exact Control}

In the following, we want to establish an exact controllability result by the construction of a particular linear, continuous and surjective operator. Indeed, we want to solve the following problem:

for all $y_{0}$ in a space to be determined after, find $\beta \in L^{2}(] 0, T[)$ such that if $y$ is a solution of the homogeneous heat equation:

$$
\left\{\begin{array}{l}
\left.y^{\prime}-\Delta y=0 \text { in } Q_{T}=\right] 0,+\infty[\times I \\
y(t, 0)=0, \quad y(t, 1)=\beta(t) \\
y(0)=y_{0} \text { in } I
\end{array}\right.
$$

then $y(T)=0$. 
Thus, considering that $y(t, 1)=\beta(t)$ and either $\mu(x)=x$, we have

$$
\left\{\begin{array}{l}
-\frac{\mathrm{d}^{2}}{\mathrm{~d} x^{2}} \mu(x)=0 \\
\mu(0)=0, \mu(1)=1
\end{array}\right.
$$

and $\mu \in L^{2}(I)$ so we get $\gamma y(t)=\beta(t) \mu$ on $] 0, T[\times \partial I$.

One can formally also see how the operator $\mathrm{L}$ can be constructed.

Multiply the Equation (12) by $\psi^{-}$solution of the Equation (11), we have:

$$
\int_{\Omega} y(T) \psi^{-}(T) \mathrm{d} x-\left\langle y_{0}, \psi_{0}\right\rangle+\int_{0}^{T} \int_{\partial I} \beta(t) \mu(\sigma) \frac{\partial \psi^{-}(t, \sigma)}{\partial v} \mathrm{~d} \sigma \mathrm{d} t=0
$$

Let

$$
\left\langle y_{0}, \psi_{0}\right\rangle=\int_{0}^{T} \int_{\partial I} \beta(t) \mu(\sigma) \frac{\partial \psi^{-}(t, \sigma)}{\partial v} \mathrm{~d} \sigma \mathrm{d} t
$$

In order for the second member to make sense, it must be assumed that: $\frac{\partial \psi^{-}(t, \sigma)}{\partial v} \in L^{2}(] 0, T[, \partial I)$. It suffices to assume that $\psi^{-} \in L^{2}(] 0, T[, D(A))$.

We know that:

$$
\sum_{k=1}^{\infty} \int_{0}^{T} \lambda_{k}^{2} \psi_{0 k}^{2} \mathrm{e}^{2 \lambda_{k} t} \mathrm{~d} t:=\sum_{k=1}^{\infty} \frac{1}{2} \lambda_{k} \psi_{0 k}^{2}\left[\mathrm{e}^{2 \lambda_{k} T}-1\right],
$$

Therefore, we deduce that

$$
\sum_{k=1}^{\infty} \int_{0}^{T} \lambda_{k}^{2} \psi_{0 k}^{2} \mathrm{e}^{2 \lambda_{k} t} \mathrm{~d} t \leq \sum_{k=1}^{\infty} \frac{1}{2} \lambda_{k} \psi_{0 k}^{2} \mathrm{e}^{2 \lambda_{k} T}<+\infty .
$$

Remember that we had defined the following spaces:

$$
G_{T}=\left\{\mu=\sum_{k=1}^{\infty} \mu_{k} w_{k} / \sum_{k=1}^{\infty} \lambda_{k} \mu_{k}^{2} \mathrm{e}^{2 \lambda_{k} T}<+\infty\right\}
$$

and we equip it with the following scalar product:

$$
(x, y)_{G_{T}}=\sum_{k=1}^{+\infty} \lambda_{k} x_{k} y_{k} \mathrm{e}^{2 \lambda_{k} T}
$$

and the natural norm $\|\cdot\|_{G_{T}}$ of dual $G_{T}^{*}$ defined by

$$
G_{T}^{*}=\left\{\mu=\sum_{k=1}^{\infty} \mu_{k} w_{k} / \sum_{k=1}^{\infty} \lambda_{k} \mu_{k}^{2} \mathrm{e}^{-2 \lambda_{k} T}<+\infty\right\}
$$

also provided with the scalar product $(,)_{G_{T}^{*}}$ and its natural norm.

With the previous notations, the formula (15) is written

$$
\begin{aligned}
\left\langle y_{0}, \psi_{0}\right\rangle_{G_{T}^{*}, G_{T}} & =\int_{0}^{T} \int_{\partial I} \beta(t) \mu(\sigma) \frac{\partial \psi^{-}(t, \sigma)}{\partial v} \mathrm{~d} \sigma \mathrm{d} t \\
& =\int_{0}^{T} \beta(t) \int_{\partial I} \mu(\sigma) \frac{\partial \psi^{-}(t, \sigma)}{\partial v} \mathrm{~d} \sigma \mathrm{d} t
\end{aligned}
$$

By integrating by part by Green, we obtain

$$
\left\langle y_{0}, \psi_{0}\right\rangle_{G_{T}^{*}, G_{T}}=\int_{0}^{T} \beta(t) \int_{I} \Delta \psi^{-} \mu \mathrm{d} x \mathrm{~d} t
$$




$$
\begin{aligned}
& =-\int_{0}^{T} \beta(t) \int_{I} \psi^{{ }^{\prime}} \mu \mathrm{d} x \mathrm{~d} t \\
& =-\int_{0}^{T} \beta(t)\left\langle\mu^{-^{\prime}}, \psi_{0}\right\rangle_{G_{T}^{*}, G_{T}} \mathrm{~d} t \\
& =-\left\langle\int_{0}^{T} \beta(t) \mu^{-^{\prime}} \mathrm{d} t, \psi_{0}\right\rangle_{G_{T}^{*}, G_{T}} .
\end{aligned}
$$

where

$$
y_{0}=-\int_{0}^{T} \beta(t) \mu^{-\prime} \mathrm{d} t \text { in } G_{T}^{*}
$$

which allows us to define the operator

$$
L_{1}(\beta)=-\int_{0}^{T} \beta(t) \mu^{-^{\prime}} \mathrm{d} t \text { in } G_{T}^{*}
$$

Remark. We can notice that: $x=y$ in $G_{T}^{*}$ equals

$$
(-\Delta)^{-1} x^{+}(T)=(-\Delta)^{-1} y^{+}(T) \text { in } L^{2}(I)
$$

We thus define the operator L by

$$
L(\beta)=\int_{0}^{T} \beta(t)(-\Delta)^{-1} \mu^{+^{\prime}}(T-t) \mathrm{d} t
$$

Using the relationship

$$
\left.\mu^{+^{\prime}}(T-t)-\Delta \mu^{+}(T-t)=0 \text { in }\right] 0, T[\times I
$$

Remark. The Lemma 1 and Lemma 2, responds to another philosophy than the usual one whose main hypothesis is the coercivity that assumes the verification of an estimate difficult to establish in explicit spaces.

So, we have the following boundary controllability result

Theorem 4 (Main result). For all $y_{0} \in G_{T}^{*}$, it exists $\beta \in L^{2}(] 0, T[)$ such that if $y$ is the solution of

$$
\left\{\begin{array}{l}
\left.y^{\prime}-\Delta y=0 \text { in } Q_{T}=\right] 0,+\infty[\times I \\
y(t, 0)=0, \quad y(t, 1)=\beta(t) \\
y(0)=y_{0} \text { in } I
\end{array}\right.
$$

then $y(T)=0$.

Proof. First step:

By construction, $L \in \mathcal{L}\left(L^{2}(] 0, T[), L^{2}(I)\right)$. Indeed, the operator $L$ is exactly defined by a formula (26); So just show that $\mu$ is strategic.

We have $\mu \in L^{2}(I)$, after that

$$
\begin{aligned}
\mu_{k} & =\sqrt{2} \int_{0}^{1} x \sin (k \pi x) \mathrm{d} x, \quad \forall k \geq 1 \\
& =-\frac{\sqrt{2}}{k \pi}(-1)^{k}+\left.\frac{\sqrt{2}}{k \pi} \sin (k \pi x)\right|_{0} ^{1} \\
& =-\sqrt{2} \frac{(-1)^{k}}{k \pi} \quad \forall k \geq 1
\end{aligned}
$$

So $\mu$ is strategic: not degenerated.

\section{Second step:}

Let's show that $L \in \mathcal{L}\left(L^{2}(] 0, T[), L^{2}(I)\right)$ is surjective? 
We know that the operator $L$ is defined by:

$$
\begin{aligned}
L(\beta) & =\int_{0}^{T} \beta(t)(-\Delta)^{-1} \mu^{+^{\prime}}(T-t) \mathrm{d} t \\
& =\int_{0}^{T} \beta(t) \sum_{k=1}^{+\infty} \mu_{k} \mathrm{e}^{-\lambda_{k}(T-t)} w_{k} \mathrm{~d} t \\
& =\int_{0}^{T} \sum_{k=1}^{\infty} \beta(t) \mu_{k} \mathrm{e}^{-\lambda_{k}(T-t)} w_{k} \mathrm{~d} t
\end{aligned}
$$

and his dual is

$$
\begin{aligned}
L^{*}(\beta) & =\int_{0}^{T} \sum_{k=1}^{\infty} \beta(t) \mu_{k} \mathrm{e}^{-\lambda_{k}(t-T)} w_{k} \mathrm{~d} t \\
& =\int_{0}^{T} \sum_{k=1}^{\infty} \beta(t) \mu_{k} \mathrm{e}^{\lambda_{k}(T-t)} w_{k} \mathrm{~d} t
\end{aligned}
$$

So

$$
\begin{aligned}
\left\|L^{*}(\beta)\right\|_{G_{T}^{*}}^{2} & \geq \int_{0}^{T} \sum_{k=1}^{\infty}\|\beta(t)\|^{2}\left\|\mu_{k}\right\|^{2} \mathrm{e}^{-2 \lambda_{k}(T-t)}\left\|w_{k}\right\|^{2} \mathrm{~d} t \\
& \geq\|\beta\|_{L^{2}(] 0, T[)}^{2}\left\|\mu_{k}\right\|^{2} \mathrm{e}^{-2 \lambda_{k}(T-t)}\left\|w_{k}\right\|^{2} \\
& \geq K \cdot\|\beta\|_{L^{2}(] 0, T[)}^{2}
\end{aligned}
$$

where $K$ a constant defined by $K=\min _{\{k \geq 1\}}\left\|\mu_{k}\right\|^{2} \mathrm{e}^{-2 \lambda_{k}(T-t)}\left\|w_{k}\right\|^{2}$.

By the Lemma 1 and Lemma 2, we can deduce that $L$ is surjective.

\section{Third step: conclusion.}

We know that $\mu$ is not degenerate, the operator $L$ is surjective and, in addition, the operator $L L^{*}$ is compact see also Seck.

Let now $y_{0} \in G_{T}^{*}$, it exists $\beta \in L^{2}(\Omega)$ such that

$$
(-\Delta)^{-1} y^{+}(T)=-\int_{0}^{T} \beta(t) \mu^{+}(T-t) \mathrm{d} t
$$

so

$$
y^{+}(T)=-\int_{0}^{T} \beta(t)(-\Delta) \mu^{+}(T-t) \mathrm{d} t
$$

Let

$$
y^{+}(T)=-\int_{0}^{T} \beta(t) \mu^{+^{\prime}}(T-t) \mathrm{d} t \text { so } y_{0}=-\int_{0}^{T} \beta(t) \mu^{-^{\prime}}(t) \mathrm{d} t
$$

Let $\psi_{0} \in G_{T}$, we have

$$
\left\langle y_{0}, \psi_{0}\right\rangle_{G_{T}^{*}, G_{T}}=\sum_{k=1}^{+\infty} \int_{0}^{T} \beta(t) \mu_{k} \mathrm{e}^{\lambda_{k} t} \lambda_{k} \psi_{0 k} \mathrm{~d} t
$$

As

$$
\begin{aligned}
& \sum_{k=1}^{+\infty} \int_{0}^{T}\left|\beta(t) \| \mu_{k}\right|\left|\lambda_{k}\right| \psi_{0 k} \mathrm{e}^{\lambda_{k} t} \mathrm{~d} t \\
& \leq \frac{1}{\sqrt{2}} \sum_{k=1}^{\infty}\|\beta\|_{L^{2}(] 0, T[)}\left|\mu_{k}\right| \lambda_{k}\left|\psi_{0 k}\right| \mathrm{e}^{\lambda_{k} T} \\
& \leq \frac{1}{\sqrt{2}}\|\beta\|_{L^{2}(] 0, T[)}\|\mu\|_{L^{2}(] 0, T[)}\left\|\psi_{0}\right\|_{G_{T}}
\end{aligned}
$$


So,

$$
\begin{aligned}
\left\langle y_{0}, \psi_{0}\right\rangle_{G_{T}^{*}, G_{T}} & =-\int_{0}^{T} \beta(t) \sum_{k=1}^{+\infty} \mu_{k} \mathrm{e}^{\lambda_{k} t} \lambda_{k} \psi_{0 k} \mathrm{~d} t \\
& =\int_{0}^{T} \beta(t) \int_{I} \mu \psi^{-1}(t) \mathrm{d} x \mathrm{~d} t \\
& =\int_{0}^{T} \beta(t) \int_{I} \mu \Delta \psi^{-}(t) \mathrm{d} x \mathrm{~d} t \\
& =\int_{0}^{T} \int_{\partial I} \frac{\partial \psi^{-}(t, \sigma)}{\partial v} \mu \mathrm{d} \sigma \mathrm{d} t
\end{aligned}
$$

Now, multiply the system (27) by $\psi^{-}(t)$ integrating by parts

$$
\left\langle y(T), \psi^{-}(T)\right\rangle_{H^{-1}(I), H_{0}^{1}(I)}-\left\langle y_{0}, \psi_{0}\right\rangle_{G_{T}^{*}, G_{T}}=-\int_{0}^{T} \beta(t) \frac{\partial \psi^{-}(t, 1)}{\partial v} \mathrm{~d} t
$$

So

$$
\forall \psi_{0} \in G_{T},\left\langle y(T), \psi^{-}(T)\right\rangle_{H^{-1}(I), H_{0}^{1}(I)}=0
$$

From where $y(T)=0:$ which completes the proof.

\section{Conclusion and Perspective}

The exact controllability results by the HUM (Hilbert Uniqueness Method of Lions) method are not suitable for parabolic type operators. To get around these difficulties, in particular the coercivity hypothesis for the establishment of the inverse inequality, many have used Carlemann inequalities Fursikov-Imanuvilov, Lebeau-Robbiano, Khodja [13], Tusnack [14], ... But the length and heaviness of the calculations in the Carleman inequalities are dissuasive, from where this idea came to us to couple the notion of strategic zone actuators and the subjectivity-compactness of a linear operator which allowed us to have the exact controllability. And this technique opens up many perspectives for linear and semi-linear parabolic systems, of Schrodinger, of plates, ...

\section{Acknowledgements}

- The authors thank the referees in advance for their comments and suggestions.

- The authors thank the Dean of FASTEF ex ENS of the University Cheikh Anta Diop in Dakar and his assessor for their financial and moral support.

\section{Conflicts of Interest}

The authors declare no conflicts of interest regarding the publication of this paper.

\section{References}

[1] Lions, J.-L. (1988) Contrôlabilité exacte, perturbations et stabilisation de systèmes distribués. Tome 2, Recherches en Mathématiques Appliquées [Research in Applied Mathematics], Volume 9, Perturbations, Masson, Paris.

[2] Lions, J.-L. (1988) Contrôlabilité exacte, perturbations et stabilisation de systèmes 
distribués. Tome 1, Recherches en Mathématiques Appliquées [Research in Applied Mathematics], Volume 8, Paris.

[3] Lions, J.-L. and Magenes, E. (1968) Problèmes aux limites non homogènes et applications. Paris, Dunod.

[4] Niane M.T. (1990) Régularité, contrôlabilité exacte et contrôlabilité spectrale de l'équation des ondes et de l'équation des plaques vibrantes. Thèse de Doctorat d'Etat, Université Cheikh Anta Diop de Dakar, Sénégal.

[5] Seck, C. (2019) Exact Controllability of the Heat Equation by Temporarily Strategic Actuators Borders. Journal of Mathematical Research, 11, 53-57. https://doi.org/10.5539/jmr.v11n6p53

[6] Seck, C. (2019) Exact Controllabilty of the Heat Equations in Any Dimension by Actuators Strategic Areas.

[7] Fursikov, A.V. and Imanuvilov, O.Yu. (1996) Controllability of Evolution Equations. Séoul National University, Séoul.

[8] Lebeau, G. and Robbiano, L. (1994) Contrôle exact de l'équation de la Chaleur. Prépublications Univ. Paris-Sud.

[9] Karim, R. (2013) Frequency and Spectral Approaches for the Control of Conservative Linear Systems in Infinite Dimension, TIPE Days.

[10] El Jai, A. (2002) Analyse régionale des systèmes distribués. ESAIM Control, Optimisation and Calculus of Variation, 8, 663-692. https://doi.org/10.1051/cocv:2002054

[11] El Jai A. (2003) Quelques problèmes de contrôle propres aux systèms distributes. Annals of University of Craiova, Math. Comp. Sci. Ser., Vol. 30, 137-153.

[12] Haim, B. (1983) Analyse fonctionnelle, Théorie et applications, Masson.

[13] Khodja, F.A., De Teresa, L., Benabdallah, A. and Gonzlez-Burgos, M. (2014) Minimal Time for the Null Controllability of Parabolic Systems: The Effect of the Condensation Index of Complex Sequences. Journal of Functional Analysis, 267, 2077-2151. https://doi.org/10.1016/j.jfa.2014.07.024

[14] Tucsnak, M. and Tenenbaum, G. (2007) New Blow-Up Rates for Fast Controls of Schrdinger and Heat Equations. Journal of Differential Equations, 243, 70-100. https://doi.org/10.1016/j.jde.2007.06.019 